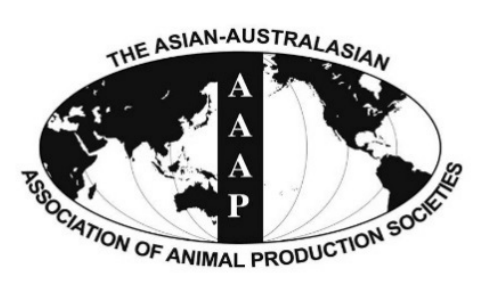

Open Access

Asian Australas. J. Anim. Sci.

Vol. 29, No. 3 : 352-358 March 2016

http://dx.doi.org/10.5713/ajas.15.0341

www.ajas.info

pISSN 1011-2367 elSSN 1976-5517

\title{
Effect of Antioxidant Flavonoids (Quercetin and Taxifolin) on In vitro Maturation of Porcine Oocytes
}

\author{
Jung-Taek Kang, Joon Ho Moon, Ji-Yei Choi, Sol Ji Park, Su Jin Kim, \\ Islam M. Saadeldin ${ }^{1}$, and Byeong Chun Lee* \\ Department of Theriogenology and Biotechnology, College of Veterinary Medicine, \\ Seoul National University, Seoul 08826, Korea
}

\begin{abstract}
Quercetin (QT) and taxifolin (TF) are structurally similar plant-derived flavonoids that have antioxidant properties and act as free radical scavengers. The objective of this study was to investigate effects of QT and TF on nuclear maturation of porcine oocytes. Effects of TF at $0,1,10$, and $50 \mu \mathrm{g} / \mathrm{mL}$ on oocyte nuclear maturation (polar body extrusion) were investigated. After incubation for $44 \mathrm{~h}$, there were no significant differences between the treatment and control groups except in the $50 \mu \mathrm{g} / \mathrm{mL}$ group which was significantly lower $(59.2 \%, \mathrm{p}<0.05)$ than the other groups (control: $>80 \%$ ). After parthenogenetic activation, further in vitro development of QT- or TF-treated vs control oocytes was investigated. A significantly higher proportion of QT-treated $(1 \mu \mathrm{g} / \mathrm{mL})$ oocytes developed into blastocysts compared to controls ( $24.3 \%$ vs $16.8 \%$, respectively); however, cleavage rate and blastocyst cell number were not affected. The TF-treated group was not significantly different from controls. Levels of reactive oxygen species (ROS) and intracellular glutathione (GSH) in oocytes and embryos in a culture medium supplemented with QT or TF were measured. Both treatment groups had significantly lower $(\mathrm{p}<0.05)$ levels of ROS than controls, however GSH levels were different only in QT-treated oocytes. We conclude that exogenous flavonoids such as QT and TF reduce ROS levels in oocytes. Although at high concentration (50 $\mu \mathrm{g} / \mathrm{mL}$ ) both QT and TF appear to be toxic to oocytes. (Key Words: In vitro Maturation, Porcine Oocyte, Antioxidants, Quercetin, Reactive Oxygen Species [ROS])
\end{abstract}

\section{INTRODUCTION}

Increasing the efficiency of systems for in vitro production of porcine embryos is very important because pigs have high biomedical value for areas such as xenotransplantation and as models for stem cell research (Telugu et al., 2011). However, despite intensive efforts, the yield and quality of in vitro matured (IVM) oocytes and embryos derived from them are still low compared with in vivo produced embryos. Improvements can be made by altering the culture conditions for oocyte maturation and embryo development, including the external oxygen

\footnotetext{
* Corresponding Author: Byeong Chun Lee. Tel: +82-2-8801269, Fax: +82-2-873-1269, E-mail: bclee@snu.ac.kr

${ }^{1}$ Department of Physiology, Faculty of Veterinary Medicine, Zagazig University, 44519, Egypt.

Submitted Apr. 20, 2015; Revised Jul. 4, 2015; Accepted Jul. 17, 2015
}

concentration (Kang et al., 2012). Oxidative stress originating from high external oxygen concentration can produce reactive oxygen species (ROS), which may be responsible for damaging embryos and inducing early embryonic developmental blocks (Guerin et al., 2001). Therefore, supplementing maturation and culture media with antioxidants such as $\beta$-mercaptoethanol, cysteine and cysteamine can help to protect against defective embryo development (Abeydeera et al., 1998; de Matos and Furnus, 2000).

Flavonoids are a class of plant secondary metabolites and are most commonly known for their antioxidant activity in vitro (Bagchi et al., 1999). Quercetin (QT) (2-(3,4dihydroxyphenyl)-3,5,7-trihydroxy-4H-chromen-4-one) and taxifolin (TF) (2-(3,4-dihydroxyphenyl)-3,5,7-trihydroxy2,3-dihydrochromen-4-one) are plant-derived flavonoids mainly found in fruits and vegetables. It has been reported 
that QT and TF have anti-oxidative, anti-mutagenic and anti-inflammatory activities (Naderi et al., 2003; Tang et al., 2006; Yuan et al., 2006; Kwon et al., 2011) due to free radical scavenging. Also, $\mathrm{TF}$ is not mutagenic and has low toxicity compared to the related compound QT (Makena et al., 2009). Our previous study on porcine oocytes demonstrated that exogenous QT is beneficial for nuclear maturation during IVM and subsequent embryo development by reducing ROS levels (Kang et al., 2013).

However, little information is available on the effect of flavonoids on oocyte maturation and embryonic development in pigs. The objective of this study was to examine the effect of QT and TF treatment during IVM and in vitro culture (IVC) on oocyte maturation and the development of parthenogenetically activated (PA) embryos. To this end, we observed nuclear maturation of oocytes, embryo cleavage and blatocyst formation of PA embryos, as well as intracellular levels of glutathione (GSH) and ROS in pig oocytes and embryos.

\section{MATERIALS AND METHODS}

Unless otherwise specified, all reagents were purchased from Sigma-Aldrich (St. Louis, MO, USA).

\section{Oocyte collection and in vitro maturation}

Pig ovaries were collected from a local abattoir and transported to the laboratory in $0.9 \%(\mathrm{w} / \mathrm{v}) \mathrm{NaCl}$ solution at $25^{\circ} \mathrm{C}$ to $30^{\circ} \mathrm{C}$. Follicular contents from antral follicles (3 to $6 \mathrm{~mm}$ in diameter) were aspirated using an 18-gauge needle attached to a $10 \mathrm{~mL}$ disposable syringe. The contents were pooled in a conical tube at $39^{\circ} \mathrm{C}$ and allowed to settle for a few minutes. The sediment was aspirated and diluted with Dulbecco's phosphate buffered saline (D-PBS; Invitrogen, Carlsbad, CA, USA) containing $100 \mathrm{U} / \mathrm{mL}$ penicillin $\mathrm{G}$ and $100 \mathrm{mg} / \mathrm{mL}$ streptomycin sulphate (pen-strep; Invitrogen, Carlsbad, CA, USA). Cumulus-oocyte complexes (COCs) with intact compact cumulus cell layers were selected and washed 3 times in tissue culture medium (TCM)-Hepes before being transferred to a modified TCM-199 supplemented with $10 \mathrm{ng} / \mathrm{mL}$ epidermal growth factor, 0.57 $\mathrm{mM}$ cystine, $0.91 \mathrm{mM}$ sodium pyruvate, $5 \mu \mathrm{g} / \mathrm{mL}$ insulin, $1 \%(\mathrm{v} / \mathrm{v})$ pen-strep, $0.5 \mu \mathrm{g} / \mathrm{mL}$ follicle stimulating hormone, $0.5 \mu \mathrm{g} / \mathrm{mL}$ luteinizing hormone and $10 \%$ porcine follicular fluid. For the first $22 \mathrm{~h}$ only, the IVM medium also contained Gonadotropin. The COCs were cultured at $38^{\circ} \mathrm{C}$ with $5 \% \quad \mathrm{CO}_{2}$ at maximum humidity. After $44 \mathrm{~h}$ of maturation, oocytes were denuded of cumulus cells by pipetting with $0.1 \%$ hyaluronidase in D-PBS supplemented with $0.1 \%$ polyvinyl alcohol. Then denuded oocytes were treated according to the experimental design.

\section{Assessment of meiotic maturation of mature oocytes}

After culture for $44 \mathrm{~h}$, denuded oocytes were stained with Hoechst 33342 in D-PBS. The stage of meiotic maturation was determined by examination of the presence or absence of the first polar body (metaphase II) under UV light.

\section{Parthenogenetic activation of mature oocytes and in vitro culture}

At 44 hours of IVM, oocytes in metaphase II were activated. Denuded oocytes were equilibrated for $1 \mathrm{~min}$ in $0.26 \mathrm{M}$ D-mannitol-based activation solution, supplemented with $0.1 \mathrm{mM} \mathrm{MgCl}_{2}, 0.1 \mathrm{mM} \mathrm{CaCl}$ and $0.5 \mathrm{mM}$ Hepes, then transferred to a chamber between two electrodes spaced $3.2 \mathrm{~mm}$ apart and overlaid with activation solution. The oocytes were activated with an electric stimulus using a single direct current pulse of $1.5 \mathrm{kV} / \mathrm{cm}$ for $60 \mu \mathrm{sec}$ using a BTX Electro-Cell Manipulator 2001 (BTX Inc., Holliston, MA, USA). A group of approximately 20 to 30 parthenogenetically-activated (PA) oocytes were cultured in $500 \mu \mathrm{L}$ porcine zygote medium (PZM)-3 containing $0.3 \%$ $(\mathrm{w} / \mathrm{v})$ fatty acid-free bovine serum albumin for 7 days at $39^{\circ} \mathrm{C}$ in a humidified atmosphere of $5 \% \mathrm{CO}_{2}$ and $5 \% \mathrm{O}_{2}$. Cleavage and blastocyst formation rates were checked at 48 and 168 hours of IVC, respectively.

\section{Assessment of embryo quality}

The quality of blastocysts was assessed by Hoechst staining of total cell number including inner cell mass and trophectoderm cells according to standard procedures. Briefly, after rinsing in PZM-3 medium, blastocysts were stained with Hoechst 33342 for 15 min. After rinsing in PZM-3 medium, the blastocysts were mounted on a glass slide under a cover slip and examined under an inverted microscope (Nikon Corp., Tokyo, Japan) equipped with epifluorescence.

\section{Measurement of intracellular glutathione and reactive oxygen species levels}

Oocytes were sampled after $44 \mathrm{~h}$ of IVM and $2 \mathrm{~d}$ of IVC to determine intracellular GSH and ROS levels using the dichlorohydrofluorescein diacetate (DCHFDA) and CellTracker Blue CMF2HC (4-chloromethyl-6,8-difluoro7-hydroxycoumarin) methods previously described (Yang et al., 1998; You et al., 2010) with slight modification. Briefly, 20 oocytes and embryos from each treatment group were incubated in the dark at $39^{\circ} \mathrm{C}$ for $30 \mathrm{~min}$ in Tyrode's albumin lactate pyruvate (TALP) medium supplemented with $10 \mu \mathrm{M}$ DCHFDA and $10 \mu \mathrm{M}$ CellTracker. After incubation, oocytes were washed in Hepes-buffered TALP medium, placed on a glass slide and covered with a cover slip. The fluorescence emissions from the oocytes and 
embryos were recorded as TIFF files using a cooled chargecoupled device (CCD) camera attached to a fluorescence microscope (Axio Photo; Carl Zeiss Jena GmbH, Berlin, Germany) with excitation filters (460 $\mathrm{nm}$ for ROS and 370 $\mathrm{nm}$ for GSH). The recorded fluorescent images were analyzed using NIH image software 1.55 (National Institutes of Health, USA) by counting the number of pixels after color inversion (Kim et al., 2006).

\section{Effect of flavonoid on cumulus cell steroidogenesis}

IVM media of both the first and second day of culture were collected, centrifuged at 1,500 rpm for $3 \mathrm{~min}$ and the supernatants were stored at $-20^{\circ} \mathrm{C}$ until assayed for progesterone (P4) and estradiol $-17 \beta$ (E2) by validated redioimmunoassays. All samples were analyzed with assistance of the Neodin Veterinary Laboratory (Seoul, Repulic of Korea; http://www.vetlab.co.kr).

\section{Experimental design}

In order to determine effective concentrations of TF for improving oocyte maturation (Experiment 1), it was included in the IVM medium (TCM-199) at four concentrations $(0,1,10$, or $50 \mu \mathrm{g} / \mathrm{mL})$ during the entire maturation culture period of $44 \mathrm{~h}$. In Experiment 2, we evaluated the effects of including QT or TF in the IVM medium compared with a non-treated control group on the parthenogenetic development of embryos. In Experiment 3, we assessed the effects of these QT and TF concentrations in the IVM medium on the ROS and GSH levels in oocytes and embryos.

\section{Statistical analysis}

All statistical analyses were performed using Prism4 software (GraphPad, La Jolla, CA, USA). One-way analysis of variance was used to determine significant differences in the data followed by a Tukey test to determine statistical differences among groups. Significant differences among the treatments were determined when $p<0.05$. Data are expressed as means \pm standard error of the mean.
Table 1. Effects of taxifolin during oocyte IVM on the frequency of first polar body extrusion (nuclear maturation)

\begin{tabular}{lccc}
\hline $\begin{array}{c}\text { Concentration } \\
(\mu \mathrm{g} / \mathrm{mL})\end{array}$ & Oocytes $(\mathrm{n})$ & $\begin{array}{c}\text { Oocytes with } \\
\text { polar body } \\
\text { extrusion }(\mathrm{n})\end{array}$ & $\begin{array}{c}\text { Polar body } \\
\text { extrusion rate } \\
(\% \pm \mathrm{SEM})\end{array}$ \\
\hline $0($ Control $)$ & 181 & 146 & $80.54 \pm 3.5^{\mathrm{a}}$ \\
1 & 192 & 162 & $84.43 \pm 2.6^{\mathrm{a}}$ \\
10 & 194 & 152 & $78.50 \pm 3.2^{\mathrm{a}}$ \\
50 & 179 & 107 & $59.21 \pm 7.9^{\mathrm{b}}$ \\
\hline
\end{tabular}

IVM, in vitro matured; SEM, standard error of the mean.

Polar bodies were counted by Hoechst staining after $44 \mathrm{~h}$ maturation in vitro.

${ }^{a, b}$ Within a column, values with different superscripts are significantly different $(\mathrm{p}<0.05)$.

\section{RESULTS}

\section{Effect of TF on porcine oocyte nuclear maturation}

A total of 746 oocytes were used in five replicates to evaluate the effects of TF on nuclear maturation during IVM. The polar body extrusion rate was not significantly different among the control and the treatment groups at 1 or $10 \mu \mathrm{g} / \mathrm{mL}$, but it was significantly lower $(\mathrm{p}<0.05)$ in the group containing $50 \mu \mathrm{g} / \mathrm{mL}$ TF $(59.2 \% \pm 7.9 \%)$ compared with the control $(80.5 \% \pm 3.5 \%)$ and the other treatment groups (Table 1).

\section{Effects of QT and TF on PA embryo development}

Based on the results from Experiment 1 and our previous study (Kang et al., 2013), $1 \mu \mathrm{g} / \mathrm{mL}$ of QT or TF were used in Experiment 2. A total of 800 oocytes that underwent IVM in media supplemented with QT or TF were PA in nine replicates. QT or TF had no effect on the first cleavage frequency or the cell number per blastocyst (Table 2). However, a significantly greater $(p<0.05)$ proportion of blastocysts developed from oocytes when the IVM medium was supplemented with $1 \mu \mathrm{g} / \mathrm{mL}$ QT (Table 2). Further treatment with QT or TF during IVC did not have any stimulatory effect on embryonic development (data not shown).

Table 2. Effects of QT or TF treatment of porcine oocytes on subsequent development in vitro

\begin{tabular}{lcccc}
\hline $\begin{array}{c}\text { Treatment } \\
(1 \mu \mathrm{g} / \mathrm{mL})\end{array}$ & Oocytes examined, $\mathrm{n}$ & $\begin{array}{c}\text { Cleavage } \\
\mathrm{n}(\%)^{*} \\
\geq 2 \text { cell }\end{array}$ & $\begin{array}{c}\text { Blastocysts } \\
\mathrm{n} \\
(\% \pm \mathrm{SEM})\end{array}$ & $\begin{array}{c}\text { No. of cells per blastocyst } \\
(\mathrm{means} \pm \mathrm{SEM})\end{array}$ \\
\hline Control & 265 & $182(68.80)$ & 44 & $49.75 \pm 3.7$ \\
QT & 266 & $188(70.87)$ & $\begin{array}{c}(16.84 \pm 2.0)^{\mathrm{a}} \\
64\end{array}$ & $48.00 \pm 2.6$ \\
TF & 269 & $198(73.58)$ & $\begin{array}{c}(24.30 \pm 2.3)^{\mathrm{b}} \\
47\end{array}$ & $51.86 \pm 4.5$ \\
\hline
\end{tabular}

QT, quercetin; TF, taxifolin; SEM, standard error of the mean.

Percentages are based on the number of oocytes examined.

${ }^{a, b}$ Within a column, values with different superscripts are significantly different $(p<0.05)$. 

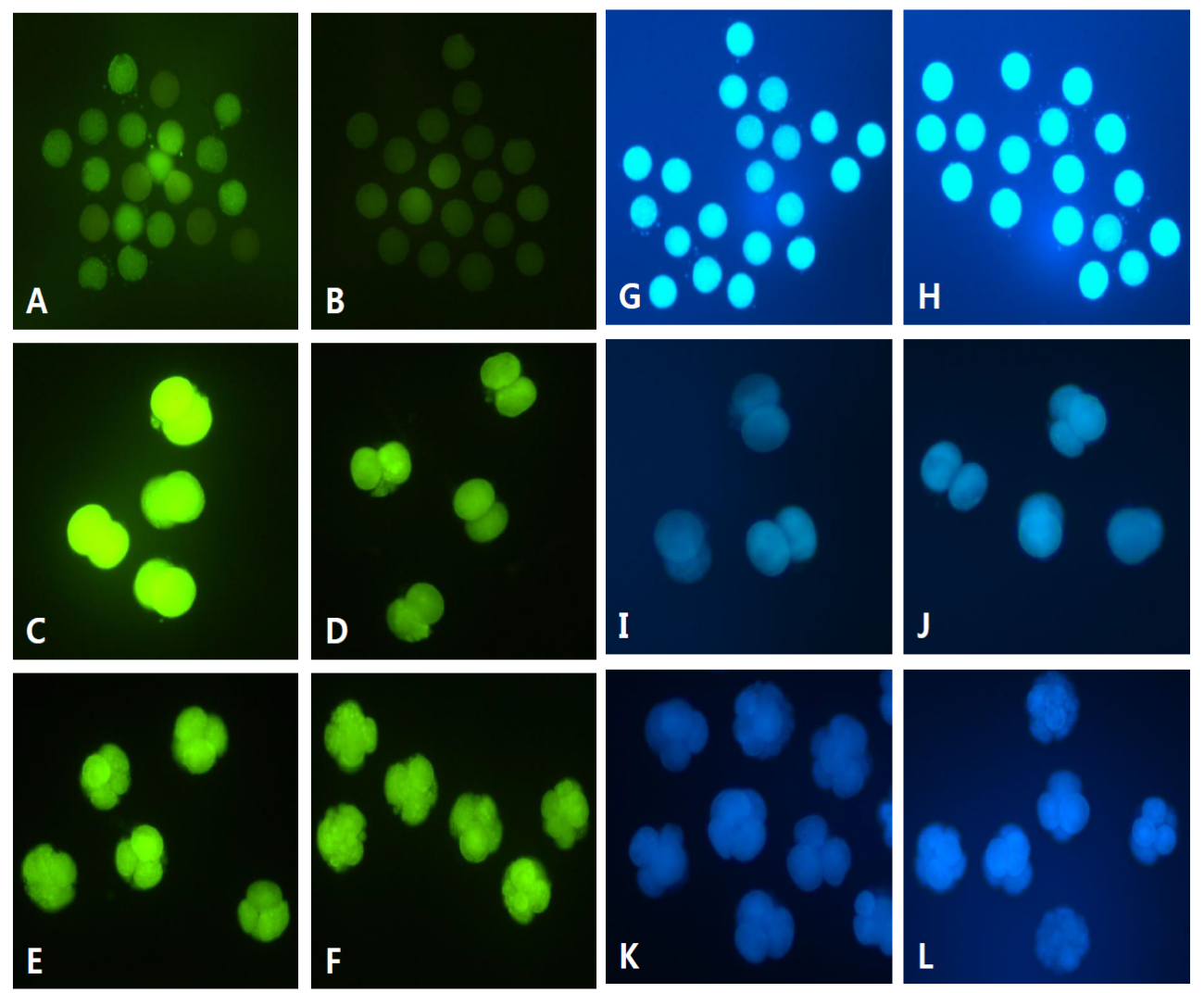

Figure 1. Epifluorescent photomicrographic images of in vitro matured porcine oocytes $(\mathrm{A}, \mathrm{B}, \mathrm{G}$, and $\mathrm{H})$ and embryos at the 2-cell $(\mathrm{C}$, $\mathrm{D}, \mathrm{I}$, and $\mathrm{J}$ ) and 4-cell stages (E, F, K, and L) developed after $2 \mathrm{~d}$ of in vitro culture. Oocytes and embryos were stained with DCHFDA (A-F) and CellTracker Blue (G-L) to detect reactive oxygen species and intracellular levels of glutathione, respectively (Original magnification $\times 200)$. Oocytes and embryos derived from the culture in medium supplemented without $(A, C, E, G, I$, and $K)$ or with $(B$, $\mathrm{D}, \mathrm{F}, \mathrm{H}, \mathrm{J}$, and L) $1 \mu \mathrm{g} / \mathrm{mL}$ quercetin.

\section{Intracellular levels of ROS and GSH in porcine oocytes} and embryos

In Experiment 3, 430 oocytes were used in four replicates to evaluate the effect of QT or TF during IVM and IVC on the levels of ROS and GSH. QT increased intracellular GSH levels and decreased ROS generation in mature oocytes and in cultured embryos $(\mathrm{p}<0.05)$. TF also reduced the ROS levels in mature oocytes $(p<0.05)$ but not in cleavage stage embryos, while GSH levels were not significantly different in oocytes or embryos compared with the control group (Figures 1 and 2).

\section{Effect of flavonoid on cumulus cell steroidogenesis}

Basal steroid production by cumulus cells after 22 and $44 \mathrm{~h}$ of culture is shown in Table 3.

Progesterone and estradiol levels in culture media of oocytes incubated with and without flavonoid (QT or TF) were investigated. Both concentration of flavonoid (QT and TF) didn't significantly have no differences.

\section{DISCUSSION}

In this study, effects of the antioxidants QT and TF were examined during oocyte maturation and embryonic development following PA, and on intracellular levels of ROS and GSH. Although porcine PA embryos cannot develop beyond 29 days of gestation in vivo (Kure-bayashi et al., 2000), they may be good models to evaluate effects of exogenous factors during in vitro embryonic development. Our results showed that the rate of PA blastocyst formation from QT-treated oocytes was significantly higher than in the control group and in the TF-treated group. However, QT did improve the cleavage rate or total cell number of blastocysts compared with the other groups. Supplementing the IVM medium with TF did not improve nuclear maturation, but was effective in reducing ROS levels in mature oocytes. The inclusion of QT but not TF in the IVM medium increased PA blastocyst formation, presumably because QT reduced ROS and increased intracellular GSH more effectively than the TF treatment. However, we have found no beneficial effect of QT or TF treatment on blastocyst formation when applied only during IVC (data not shown). It is not clear whether improved embryonic development observed after treatment of oocytes with QT or TF was due to a direct action of QT or $\mathrm{TF}$ on embryos or to the reduction of ROS toxicity by increasing GSH. 
(A)

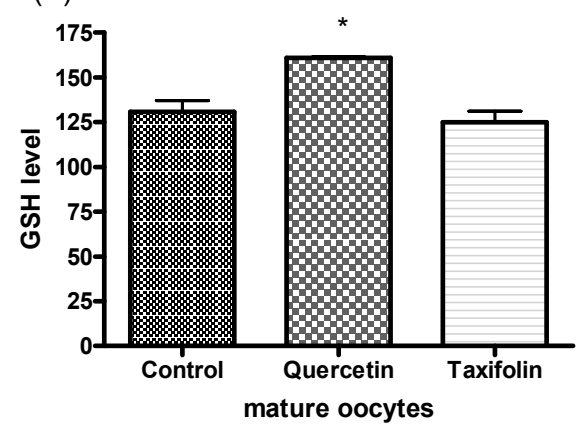

(C)

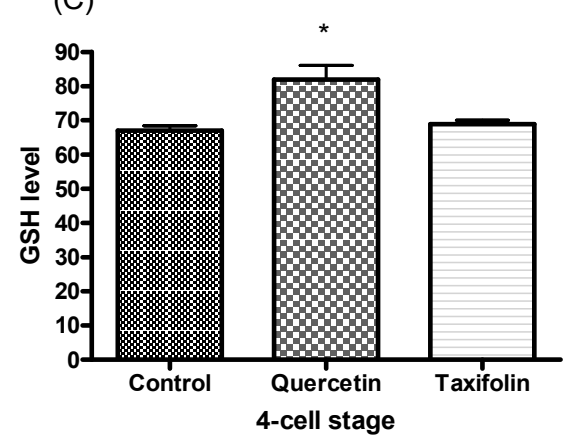

(F)

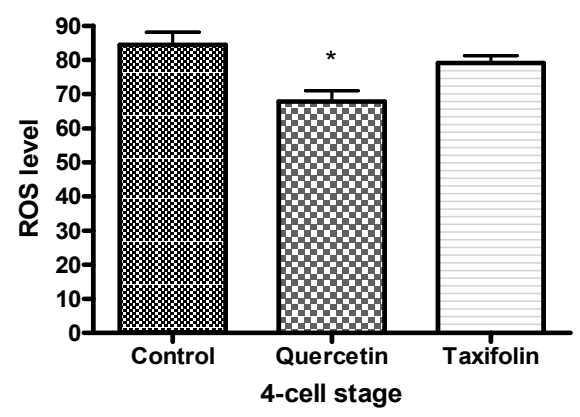

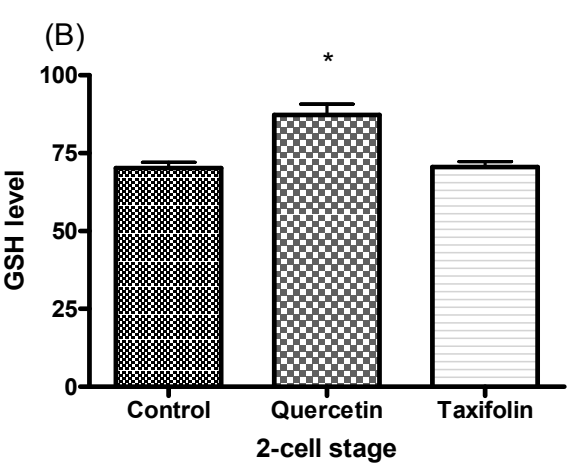

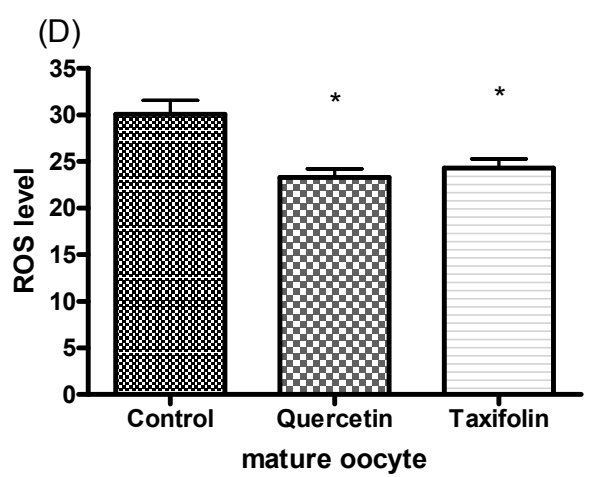

(E)

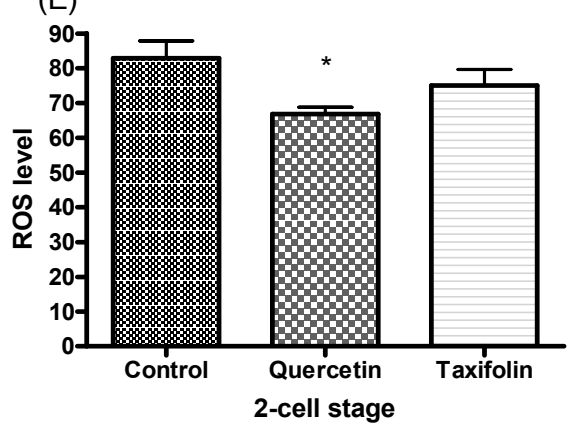

Figure 2. Measurement of intracellular glutathione (GSH) and reactive oxygen species (ROS) levels in in vitro matured (IVM) oocytes and in parthenogenetically activated embryos. The symbol $(*)$ indicates a significant difference $(\mathrm{p}<0.05)$. Values shown on the Y-axis are number of pixels.

ROS may originate from embryo metabolism and/or the embryo environment, and are detrimental to embryonic development (Johnson and Nasr-Esfahani, 1994). Many antioxidants can alleviate oxidative stress during

Table 3. Effects of flavonoid (QT and TF) on cumulus cell steroidogensesis

\begin{tabular}{lccccc}
\hline \multirow{2}{*}{ Treatment } & \multicolumn{2}{c}{ Estradiol $(\mathrm{pg} / \mathrm{mL})$} & & \multicolumn{2}{c}{ Progesterone $(\mathrm{ng} / \mathrm{mL})$} \\
\cline { 2 - 3 } \cline { 5 - 6 } & $22 \mathrm{~h}$ & $44 \mathrm{~h}$ & & $22 \mathrm{~h}$ & $44 \mathrm{~h}$ \\
\hline Control & $1,326.5$ & $1,161.7$ & & 85.0 & 323.7 \\
QT & $1,206.3$ & $1,144.3$ & & 80.7 & 295.3 \\
TF & $1,223.8$ & $1,168.0$ & & 75.8 & 395.2 \\
\hline
\end{tabular}

QT, quercetin; TF, taxifolin; E2, estradiol-17ß; P4, progesterone.

$\mathrm{E} 2$ and $\mathrm{P} 4$ production by cumulus cells after 22 and $44 \mathrm{~h}$ of in vitro maturation of oocytes in absence (control) or in presence of $1 \mu \mathrm{g} / \mathrm{mL}$ QT or $1 \mu \mathrm{g} / \mathrm{mL}$ TF. Data represent mean of at least four replicates. reproductive processes, and can enhance embryonic development in vitro. In pigs, as in other mammals, several antioxidants have been used as supplements in culture media to enhance embryonic development (Kang et al., 2009), but little research has been done with flavonoids, which are well-known and powerful natural antioxidants. To the best of our knowledge, this is the first study investigating QT or TF effects on early embryonic development in vitro in pigs. Our data indicate that the optimal amount of flavonoid is concentration-specific, and while lower concentrations elicit no observable responses, excessive levels could be toxic to the oocyte.

Due to their structural similarity with estrogen, several flavonoids, including genistein and daidzein, also interact with the estrogen receptor to mediate their activity and 
thereby act as weak or moderate phytoestrogens (Galeati et al., 2010). Female mice treated neonatally with genistein showed multi-oocyte follicles, lacked regular estrous cyclicity and showed implantation failure, although ovulated oocytes were developmentally competent (Jefferson et al., 2009). Therefore, to confirm effects of flavonoids as phytoestrogens, we assayed for P4 and E2 by radioimmunoassay during the oocyte maturation period (44 h). For P4 and E2, steroid concentrations are expressed steadily without differences among groups (control, 1 $\mu \mathrm{g} / \mathrm{mL}$ QT- and TF- treated group) (Table 3). Maybe it thought that QT and TF in this concentration is not effective in changing basal estradiol-17 $\beta$ secretion as phytoestrogens, and this finding is in agreement with the results of other flavonoids obtained by Galeati et al. (2010) in porcine granulosa cells, showing that these flavonoids in this concentration may affect very weakly on oocytes or granulosa cells as phytoestrogens.

Previous studies reported that addition of high concentrations of antioxidants to the IVM medium decreased the rate of blastocyst formation compared to treatment with low concentrations (Boquest et al., 1999), suggesting that the proper concentration of an antioxidant can contribute to the generation of high quality embryos. In our experiments, treatment with $50 \mu \mathrm{g} / \mathrm{mL}$ TF was detrimental to oocyte maturation, which is consistent with the result using QT in our previous study (Kang et al., 2013). One study reported that QT at $50 \mu \mathrm{g} / \mathrm{mL}$ inhibited progesterone production by granulosa cells, altered estradiol-17 $\beta$ production, and interfered with angiogenesis by inhibiting vascular endothelial growth factor production, so QT may have a negative influence on ovarian physiology (Santini et al., 2009). Both QT and TF in high concentration (at least $50 \mu \mathrm{g} / \mathrm{mL}$ ) may have detrimental effects to oocytes by influencing culture environments.

We compared effects of QT and its analogue TF, both at $1 \mu \mathrm{g} / \mathrm{mL}$, on embryo development after parthenogenetic activation (Experiment 2). Previous research has shown that using antioxidants during oocyte maturation increases cytoplasmic maturation and leads to higher rates of IVF and embryo development (Abeydeera et al., 1998). In our study, the antioxidant QT applied during IVM increased intracellular GSH levels and improved blastocyst development, which implies enhancement of cytoplasmic maturation. However, no beneficial effect of TF treatment during IVM was found on first polar body extrusion rate, showing that it could not affect nuclear maturation even though it effectively reduced ROS levels. Also, our observations that antioxidants, specifically QT, applied during oocyte maturation increases blastocyst formation, are in agreement with previous findings (Tao et al., 2004; Whitaker and Knight, 2004; You et al., 2010).

In conclusion, treatment of porcine oocytes with the flavonoid QT had a significant positive effect on embryonic development and reduced ROS generation by increasing intracellular GSH levels at low concentrations, but it was detrimental at high concentrations. It is not clear whether this concentration of QT is optimal in pigs. Therefore, further studies are needed to determine the optimal concentration of QT and to ascertain its beneficial effects on further development of pig embryos.

\section{CONFLICT OF INTEREST}

We certify that there is no conflict of interest with any financial organization regarding the material discussed in the manuscript.

\section{ACKNOWLEDGMENTS}

This study was supported by IPET (\#311011-05-4SB010, \#114059-03-2-SB010), MI (\#10048948), Research Institute for Veterinary Science, TS corporation and the BK21 plus program.

\section{REFERENCES}

Abeydeera, L. R., W. H. Wang, T. C. Cantley, R. S. Prather, and B. N. Day. 1998. Presence of beta-mercaptoethanol can increase the glutathione content of pig oocytes matured in vitro and the rate of blastocyst development after in vitro fertilization. Theriogenology 50:747-756.

Bagchi, D., O. R. Carryl, M. X. Tran, M. Bagchi, A. Garg, M. M. Milnes, C. B. Williams, J. Balmoori, D. J. Bagchi, S. Mitra, and S. J. Stohs. 1999. Acute and chronic stress-induced oxidative gastrointestinal mucosal injury in rats and protection by bismuth subsalicylate. Mol. Cell Biochem. 196:109-116.

Boquest, A. C., L. R. Abeydeera, W. H. Wang, and B. N. Day. 1999. Effect of adding reduced glutathione during insemination on the development of porcine embryos in vitro. Theriogenology 51:1311-1319.

de Matos, D. G. and C. C. Furnus. 2000. The importance of having high glutathione (GSH) level after bovine in vitro maturation on embryo development: Effect of beta-mercaptoethanol, cysteine and cystine. Theriogenology 53:761-771.

Galeati, G., C. Vallorani, D. Bucci, C. Bernardini, C. Tamanini, A. Parmeggiani, and M. Spinaci. 2010. Daidzein does affect progesterone secretion by pig cumulus cells but it does not impair oocytes IVM. Theriogenology 74:451-457.

Guerin, P., S. El Mouatassim, and Y. Menezo. 2001. Oxidative stress and protection against reactive oxygen species in the pre-implantation embryo and its surroundings. Hum. Reprod. Update 7:175-189.

Jefferson, W. N., E. Padilla-Banks, E. H. Goulding, S. P. Lao, R. R. Newbold, and C. J. Williams. 2009. Neonatal exposure to genistein disrupts ability of female mouse reproductive tract to support preimplantation embryo development and implantation. Biol. Reprod. 80:425-431.

Johnson, M. H. and M. H. Nasr-Esfahani. 1994. Radical solutions and cultural problems: could free oxygen radicals be responsible for the impaired development of preimplantation 
mammalian embryos in vitro? Bioessays 16:31-38.

Kang, J. T., M. Atikuzzaman, D. K. Kwon, S. J. Park, S. J. Kim, J. H. Moon, O. J. Koo, G. Jang, and B. C. Lee. 2012. Developmental competence of porcine oocytes after in vitro maturation and in vitro culture under different oxygen concentrations. Zygote 20:1-8.

Kang, J. T., O. J. Koo, D. K. Kwon, H. J. Park, G. Jang, S. K. Kang, and B. C. Lee. 2009. Effects of melatonin on in vitro maturation of porcine oocyte and expression of melatonin receptor RNA in cumulus and granulosa cells. J. Pineal Res. 46:22-28.

Kang, J. T., D. K. Kwon, S. J. Park, S. J. Kim, J. H. Moon, O. J. Koo, G. Jang, and B. C. Lee. 2013. Quercetin improves the in vitro development of porcine oocytes by decreasing reactive oxygen species levels. J. Vet. Sci. 14:15-20.

Kim, J. H., S. H. Lee, S. Kim, Y. W. Jeong, O. J. Koo, M. D. Hashem, S. M. Park, E. G. Lee, M. S. Hossein, S. K. Kang, B. C. Lee, and W. S. Hwang. 2006. Embryotrophic effects of ethylenediaminetetraacetic acid and hemoglobin on in vitro porcine embryos development. Theriogenology 66:449-455.

Kure-bayashi, S., M. Miyake, K. Okada, and S. Kato. 2000. Successful implantation of in vitro-matured, electro-activated oocytes in the pig. Theriogenology 53:1105-1119.

Kwon, J. H., S. B. Kim, K. H. Park, and M. W. Lee. 2011. Antioxidative and anti-inflammatory effects of phenolic compounds from the roots of Ulmus macrocarpa. Arch. Pharm. Res. 34:1459-1466.

Makena, P. S., S. C. Pierce, K. T. Chung and S. E. Sinclair. 2009. Comparative mutagenic effects of structurally similar flavonoids quercetin and taxifolin on tester strains Salmonella typhimurium TA102 and Escherichia coli WP-2 uvrA. Environ. Mol. Mutagen. 50:451-459.

Naderi, G. A., S. Asgary, N. Sarraf-Zadegan, and H. Shirvany. 2003. Anti-oxidant effect of flavonoids on the susceptibility of LDL oxidation. Mol. Cell Biochem. 246:193-196.
Santini, S. E., G. Basini, S. Bussolati, and F. Grasselli. 2009. The phytoestrogen quercetin impairs steroidogenesis and angiogenesis in swine granulosa cells in vitro. J. Biomed. Biotechnol. Article ID 419891.

Tang, X., C. Zhang, W. Zeng, Y. Mi, and H. Liu. 2006. Proliferating effects of the flavonoids daidzein and quercetin on cultured chicken primordial germ cells through antioxidant action. Cell Biol. Int. 30:445-451.

Tao, Y., B. Zhou, G. Xia, F. Wang, Z. Wu, and M. Fu. 2004. Exposure to L-ascorbic acid or alpha-tocopherol facilitates the development of porcine denuded oocytes from metaphase I to metaphase II and prevents cumulus cells from fragmentation. Reprod. Domest. Anim. 39:52-57.

Telugu, B. P., T. Ezashi, S. Sinha, A. P. Alexenko, L. Spate, R. S. Prather, and R. M. Roberts. 2011. Leukemia inhibitory factor (LIF)-dependent, pluripotent stem cells established from inner cell mass of porcine embryos. J. Biol. Chem. 286:28948-28953.

Whitaker, B. D. and J. W. Knight. 2004. Exogenous gammaglutamyl cycle compounds supplemented to in vitro maturation medium influence in vitro fertilization, culture, and viability parameters of porcine oocytes and embryos. Theriogenology 62:311-322.

Yang, H. W., K. J. Hwang, H. C. Kwon, H. S. Kim, K. W. Choi, and K. S. Oh. 1998. Detection of reactive oxygen species (ROS) and apoptosis in human fragmented embryos. Hum. Reprod. 13:998-1002.

You, J., J. Kim, J. Lim, and E. Lee. 2010. Anthocyanin stimulates in vitro development of cloned pig embryos by increasing the intracellular glutathione level and inhibiting reactive oxygen species. Theriogenology 74:777-785.

Yuan, Z. P., L. J. Chen, L. Y. Fan, M. H. Tang, G. L. Yang, H. S. Yang, X. B. Du, G. Q. Wang, W. X. Yao, Q. M. Zhao, B. Ye, R. Wang, P. Diao, W. Zhang, H. B. Wu, X. Zhao, and Y. Q. Wei. 2006. Liposomal quercetin efficiently suppresses growth of solid tumors in murine models. Clin. Cancer Res. 12:31933199 . 